\title{
Resource Allocation for Full-Duplex Relay-Assisted Device-to-Device Multicarrier Systems
}

\author{
Shuping Dang, Student Member, IEEE, Justin P. Coon, Senior Member, IEEE, and Gaojie Chen, Member, IEEE
}

\begin{abstract}
This paper analyzes the resource allocation problem for full-duplex relay-assisted device-to-device (D2D) multicarrier systems, where multiple D2D user groups (UGs) coexist in an underlaying manner. We formulate the optimization problem, which takes the maximization of the system throughput as an objective. Two resources, i.e. subcarrier and transmit power are considered to be appropriately allocated to UGs in order to meet the objective. The formulated problem can be independently divided into a quasiconcave problem and a mixed binary integer programming (MBIP) problem. The MBIP problem is NP-hard. Therefore, to solve this problem efficiently by standard optimization techniques, we propose an alternative algorithm, which is the linear relaxation of the MBIP problem. Then, we mathematically prove and numerically verify the equivalence of the MBIP problem and its linear relaxation. By this way, the resource allocation for UGs can be carried out by a small amount of computational overhead.
\end{abstract}

Index Terms-Relay-assisted D2D systems, resource allocation, fullduplex communications, multicarrier systems.

\section{INTRODUCTION}

$\mathbf{U}$ NDERLAY device-to-device (D2D) communications in which user pairs in close proximity communicate without going through a base station (BS), have attracted a large amount of attention in recent years due to its high power efficiency, high spectral efficiency and low transmission delay [1]. As an important application of D2D communications, relay-assisted D2D systems are frequently investigated in terms of the aspects of outage performance and resource allocation etc. [2]. However, most recent works related to relay-assisted D2D systems assume relays to be half-duplex, which will degrade the network throughput by a fraction due to the use of multiple orthogonal time or frequency slots for one complete transmission. Thanks to the state-of-theart progress in self-interference (SI) cancellation technology, e.g. compact, prototyped real-time full-duplex radios [3], [4], SI can be mitigated to the noise level, which makes full-duplex communications more applicable in many systems [5]. More specifically, fullduplex D2D communications without relay have been discussed in [6], and the effects of residual SI and spectral efficiency for full-duplex D2D communications are analyzed in [7] and [8], respectively. Following that, the analysis of outage performance and power allocation of full-duplex relay-assisted D2D systems are given in [9]-[13], but in a simplified case without considering multiple user pairs and subcarriers. Most importantly, the fullduplex D2D system model with multiple non-cooperative user

(c) 2016 IEEE. Personal use of this material is permitted. Permission from IEEE must be obtained for all other users, including reprinting/ republishing this material for advertising or promotional purposes, creating new collective works for resale or redistribution to servers or lists, or reuse of any copyrighted components of this work in other works.

This work was supported by the SEN grant (EPSRC grant number EP/N002350/1) and the grant from China Scholarship Council (No. 201508060323). The authors are with the Department of Engineering Science, University of Oxford, Parks Road, Oxford, UK, OX1 3PJ; (e-mail: \{shuping.dang, justin.coon, gaojie.chen\}@eng.ox.ac.uk). (Corresponding author: Gaojie Chen.)

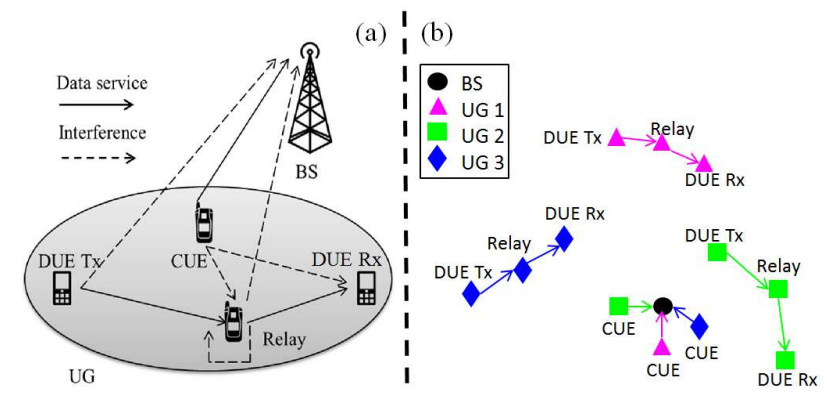

Fig. 1. (a) The transmission relations among a UG and a BS; (b) a realization of three UGs in the full-duplex relay-assisted D2D network.

groups (UGs) is proposed in [14], which paves the way for analyzing multiuser and multicarrier cases with cooperative users.

Therefore, to fill in the gap of resource allocation issues in a general and realistic scenario with multiple users and subcarriers, we analyze the resource allocation problem for full-duplex relayassisted D2D systems, where multiple UGs and subcarriers are considered. Two resources, subcarrier and transmit power are treated jointly in this paper. A resource allocation problem consisting of both is formulated and can be independently divided into a quasi-concave problem (for the allocation of transmit power) and a mixed binary integer programming (MBIP) problem (for the allocation of subcarriers). Because the MBIP problem is NP-hard [15], in order to solve this problem efficiently by standard optimization techniques, we propose an alternative allocation algorithm, in which the NP-hard MBIP problem can be approximated by a linear programming (LP) problem. Then, we also mathematically prove the equivalence of these two problems. The feasibility and efficiency of the proposed linearly relaxed allocation algorithm is subsequently verified by numerical results.

\section{System Model}

A typical UG and the fundamental transmission relations are shown in Fig. 1(a). Each UG consists of one cellular user equipment (CUE), one D2D user equipment (DUE) transmitter, one DUE receiver and one full-duplex decode-and-forward (DF) relay. Note, a UG does not necessarily imply a group of elements in close proximity, but a conceptual group of communication nodes transmitting in an underlaying manner. For illustration purposes, a realization of three UGs in the full-duplex relay-assisted D2D network is shown in Fig. 1(b) ${ }^{1}$. Without loss of generality, we assume that there exist $K$ subcarriers which can be allocated to UGs for transmissions. In order to optimize the data rate in

\footnotetext{
${ }^{1}$ There are a variety of methods to pair up a UG depending on interference and other factors [16], which are beyond the scope of this paper. Therefore, here we only adopt the concept of UG and assume all UGs are equivalent in terms of their relative locations to $\mathrm{BS}$.
} 
multiuser OFDM systems, we assume that a subcarrier should be assigned to only one UG, so that the interference among different UGs can be prevented [17]. On the other hand, it is assumed that multiple subcarriers can be allocated to and utilized simultaneously by one UG without considering the power imbalance problem. For convenience purposes, we denote the sets of UGs and subcarriers as $\mathcal{N}=\{1,2, \ldots, N\}$ and $\mathcal{K}=\{1,2, \ldots, K\}$. Meanwhile, for simplicity, we can further represent the BS, CUE, DUE transmitter, DUE receiver and relay by $B, C, S, D$ and $R$ and organize them in a set $\Theta=\{B, C, S, D, R\}$. In this paper, we adopt the Rayleigh fading model for all channels. Therefore, $\forall i \neq j$ and $i, j \in \Theta^{2}$, the channel gain between $i$ node and $j$ node for the $n$th UG and the $k$ th subcarrier, denoted as $G_{i j}(n, k)$, is assumed to be independent and non-identically exponentially distributed with average channel gain $\mu_{i j}$. Then, the PDF and CDF corresponding to each channel gain are written as

$$
f_{G_{i j}}(g)=\mu_{i j}^{-1} e^{-g / \mu_{i j}} \Leftrightarrow F_{G_{i j}}(g)=1-e^{-g / \mu_{i j}} .
$$

Meanwhile, power control is applied to all DUE transmitters and relays so that the interference from them to the BS can be mitigated. To do so, it is assumed that the power control for each subcarrier is independent and all channel state information (CSI) is known at the BS. Thus, the transmit powers of the $n$th DUE transmitter and relay over the $k$ th subcarrier are constrained by

$P_{S}(n, k)=\alpha(n, k) \eta / G_{S B}(n, k)$ and $P_{R}(n, k)=\beta(n, k) \eta / G_{R B}(n, k)$,

where $\eta$ is a predefined interference threshold at the BS, which is the same threshold for all subcarriers, due to the independent power control policy; $\alpha(n, k) \in(0,1)$ and $\beta(n, k) \in(0,1)$, satisfying $\alpha(n, k)+\beta(n, k)=1$, are the power allocation factors.

Besides, we consider an interference-limited environment, where the additive noise at receivers is negligible. As a result, the instantaneous signal-to-interference ratios (SIR) from the $n$th DUE transmitter to the $n$th relay and from the $n$th relay to the $n$th DUE receiver over the $k$ th subcarrier are written as

$$
\Gamma_{S R}(n, k)=\frac{G_{S R}(n, k) P_{S}(n, k)}{G_{C R}(n, k) P_{C}+G_{R R}(n, k)}
$$

and

$$
\Gamma_{R D}(n, k)=G_{R D}(n, k) P_{R}(n, k) /\left(G_{C D}(n, k) P_{C}\right),
$$

where $P_{C}$ is the a fixed transmit power of the CUE, which is the same for all $\mathrm{UGs}^{3}$. By adopting the DF relaying protocol and assuming the direct transmission link between DUE transmitter and DUE receiver does not exist ${ }^{4}$, therefore the equivalent end-toend instantaneous SIR and the D2D transmission rate for the $n$th user over the $k$ th subcarrier are given by

$$
\Gamma_{S R D}(n, k)=\min \left(\Gamma_{S R}(n, k), \Gamma_{R D}(n, k)\right) .
$$

and

$$
R(n, k)=\log _{2}\left(1+\Gamma_{S R D}(n, k)\right) .
$$

\footnotetext{
${ }^{2}$ An exception is given by $i=j=R$, and $G_{R R}(n, k)$ is employed to denote the instantaneous loop channel gain leading to residual SI for the $n$th UG and the $k$ th subcarrier. By SI cancellation processing, the loop channel gain $G_{R R}(n, k)$ can also be regarded as exponentially distributed.

${ }^{3}$ We do not consider the performance between the BS and CUE in this paper, and simply assume equal transmit power for all CUEs for simplicity, since by the power control specified in (2), D2D communications will not affect the cellular communications between CUE and BS.

${ }^{4}$ This common assumption is applicable to the case when the direct link cannot be established due to overlong distance between the DUE pair, and/or deep fading.
}

\section{Problem Formulation and Analysis}

From the system model built in the previous section, we can formulate the resource allocation problem as follows:

$$
\begin{array}{ll} 
& \max _{\substack{w(n), k) \\
\alpha(n, k), \beta(n, k)}} \sum_{n=1}^{N} \sum_{k=1}^{K} w(n, k) R(n, k), \quad \forall n \in \mathcal{N}, k \in \mathcal{K} \\
\text { s.t. } & w(n, k) \in\{0,1\}, \quad \sum_{n=1}^{N} w(n, k) \leq 1, \\
& \alpha(n, k) \in(0,1), \beta(n, k) \in(0,1), \alpha(n, k)+\beta(n, k)=1,
\end{array}
$$

where $w(n, k) \in\{0,1\}$ is the subcarrier allocation factor; we stipulate that $w(n, k)=1$ when the $k$ th subcarrier is allocated to the $n$th $\mathrm{UG}$ and $w(n, k)=0$ otherwise.

Due to the involvement of binary factors $w(n, k)$, the optimization problem formulated in (7) is a MBIP problem, which has been proved to be NP-hard [15]. Therefore, to solve this problem, we first divide it into two independent problems for power and subcarrier allocations, respectively. Because power and subcarrier allocations are decoupled ${ }^{5}$, regardless of the subcarrier allocation, we can first deal with the power allocation problem for the $n$th $\mathrm{UG}$ over the $k$ th subcarrier, formulated by

$$
\begin{aligned}
& \bar{R}(n, k)=\max _{\alpha(n, k), \beta(n, k)} R(n, k), \quad \forall n \in \mathcal{N}, k \in \mathcal{K} \\
& \text { s.t. } \quad \alpha(n, k) \in(0,1), \beta(n, k) \in(0,1), \alpha(n, k)+\beta(n, k)=1 .
\end{aligned}
$$

The optimization problem formulated in (8) is quasi-concave in terms of $\alpha(n, k)$ and $\beta(n, k)$ (see Appendix A for a proof), and thus the optimal power allocation factors $\alpha(n, k)$ and $\beta(n, k)$ can be efficiently determined by standard optimization techniques.

After power allocation, the subcarrier allocation problem can be written as

$$
\begin{aligned}
& \max _{w(n, k)} \sum_{n=1}^{N} \sum_{k=1}^{K} w(n, k) \bar{R}(n, k), \quad \forall n \in \mathcal{N}, k \in \mathcal{K} \\
& \text { s.t. } \quad w(n, k) \in\{0,1\} \text { and } \sum_{n=1}^{N} w(n, k) \leq 1 .
\end{aligned}
$$

As the problem formulated in (9) is still a MBIP problem and thus NP-hard, we can take a similar method as proposed in [18] by temporarily allowing allocating a subcarrier to multiple users to produce its the linear relaxation. This indicates that we can relax the value of the binary subcarrier allocation factor $w(n, k)$ to $\tilde{w}(n, k)$, which can take any real value in the interval $[0,1]$. The linear relaxation of (9) can be written as

$$
\begin{aligned}
& \max _{\tilde{w}(n, k)} \sum_{n=1}^{N} \sum_{k=1}^{K} \tilde{w}(n, k) \bar{R}(n, k), \quad \forall n \in \mathcal{N}, k \in \mathcal{K} \\
& \text { s.t. } \quad \tilde{w}(n, k) \in[0,1] \text { and } \sum_{n=1}^{N} \tilde{w}(n, k) \leq 1 .
\end{aligned}
$$

Obviously, the problem formulated in (10) is linear in terms of $\tilde{w}(n, k)$, so that can be efficiently solved by standard LP techniques. Finally, the binary subcarrier allocation factor $w(n, k)$ is quantized by the largest $\tilde{w}(n, k), \forall n \in \mathcal{N}$. By this way, the NPhard MBIP problem formulated in (9) can be approximated and solved by the LP problem formulated in (10). The equivalence of these two problems is mathematically proved in Appendix B, which indicates that the LP problem is also optimal.

\footnotetext{
${ }^{5}$ The optimization of transmission rate for all subcarrier by power allocation factors is achieved individually by each UG via the CSI related to its own.
} 


\section{NUMERICAL RESULTS}

In this section, we verify our proposed linearly relaxed allocation algorithm by Monte Carlo simulations. We set up the fixed simulation parameters as follows: $P_{C}=1$ (normalized), $\eta=2, \mu_{S B}=10 \mathrm{~dB}, \mu_{R B}=12 \mathrm{~dB}, \mu_{C R}=3 \mathrm{~dB}$ and $\mu_{C D}=2 \mathrm{~dB}^{6}$. First, we would like to show the priority of the proposed instantaneous power allocation scheme as formulated in (8) over the fixed power allocation scheme that simply specifies a fixed $\alpha$ to all UGs regardless of CSI. The subcarrier allocation applied for both is the one given in (10). To compare the instantaneous and statistical power allocation schemes, we define $\bar{\alpha}_{\Sigma}:=\mathbb{E}_{t}\left\{\sum_{n \in \mathcal{N}} \bar{\alpha}(n, k) / N\right\}$ and $a^{*}:=$ $\arg \max _{a} \mathbb{E}_{t}\left\{\left.R(n, k)\right|_{\alpha(n, k)=\alpha}\right\}^{7}$, where $\mathbb{E}_{t}\{\cdot\}$ represents the average over repeated trials and $\bar{\alpha}(n, k)$ denotes the optimized power allocation factor produced by (8). Three channel configurations for $\mu_{S R}, \mu_{R D}$ and $\mu_{R R}$ are adopted:

- Configuration 1: $\mu_{S R}=25 \mathrm{~dB}, \mu_{R D}=35 \mathrm{~dB}, \mu_{R R}=5 \mathrm{~dB}$.

- Configuration 2: $\mu_{S R}=35 \mathrm{~dB}, \mu_{R D}=25 \mathrm{~dB}, \mu_{R R}=5 \mathrm{~dB}$.

- Configuration 3: $\mu_{S R}=30 \mathrm{~dB}, \mu_{R D}=30 \mathrm{~dB}, \mu_{R R}=10 \mathrm{~dB}$.

The simulation results of average sum rate vs. $\alpha$ given $N=5$ and $K=8$ are shown in Fig. 2 , by which $\bar{\alpha}_{\Sigma}$ will converge to the optimal statistical $\alpha^{*}$ when the number of repeated trials is large, but the instantaneous power allocation scheme will produce a higher average sum rate than the statistical scheme.

Meanwhile, in order to reflect the positive impacts of resource allocation on the average sum rate and provide comparison benchmarks, we also simulate four other allocation algorithms ${ }^{8}$

- Optimal scheme: Directly apply the optimization by traversing the problem formulated in (7) with centralized coordination among all communication nodes and instantaneous CSI.

- Incomplete scheme I: Centralized coordination among all communication nodes is applied with statistical CSI.

- Incomplete scheme II: Centralized coordination among all communication nodes is applied without any CSI

- Incomplete scheme III: Instantaneous CSI is accessible by all communication nodes without a centralized coordination.

Then, the numerical results of average sum rate vs. the numbers of UGs are illustrated in Fig. 3. From this figure, the equivalence of the MBIP and LP problems can be been verified, as both the optimal and the linearly relaxed algorithms have the same average sum rate given the same system configurations. Also, it is obvious that the proposed resource allocation algorithm outperforms the three other incomplete allocation schemes.

\section{CONCLUSion}

In this paper, we formulated the resource allocation problem for full-duplex relay-assisted D2D systems and proposed an alternative allocation algorithm given by its linear relaxation. This linearly relaxed allocation algorithm has been mathematically proved and numerically verified to be equivalent to the optimal one.

\footnotetext{
${ }^{6}$ These are not necessarily fixed values if consider stochastic networks

${ }^{7}$ Because $a^{*}$ is produced by the statistical power allocation scheme which is independent from instantaneous CSI and all UGs are assumed to be equivalent, it is statistically feasible to determine $a^{*}$ via an arbitrary UG.

${ }^{8}$ These three incomplete schemes are simulated by 1) set up the optimal statistical $\alpha^{*}$ obtained by statistical power allocation for all UGs over all subcarriers and apply the linearly relaxed subcarrier allocation given in $(10)$; 2) set up random $\alpha(n, k)$ over all subcarriers and apply the linearly relaxed subcarrier allocation given in (10);3) apply the optimal power allocation scheme given in (8) with randomly allocated subcarriers to UGs.
}

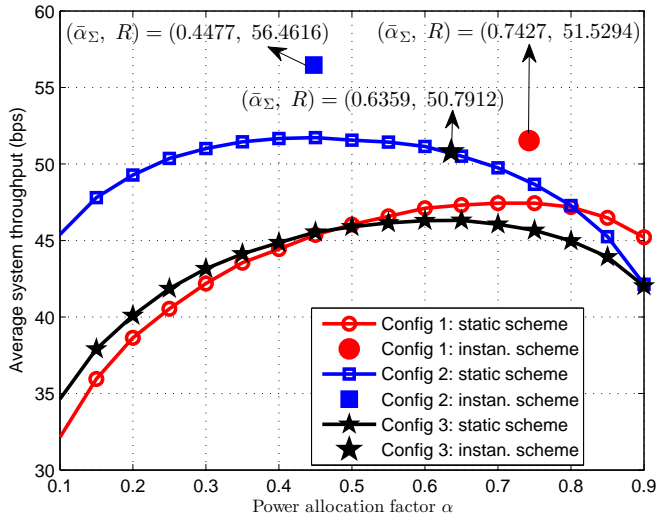

Fig. 2. Average sum rate vs statistical power allocation factor $\alpha$ for different channel configurations when $N=5$ and $K=8$.

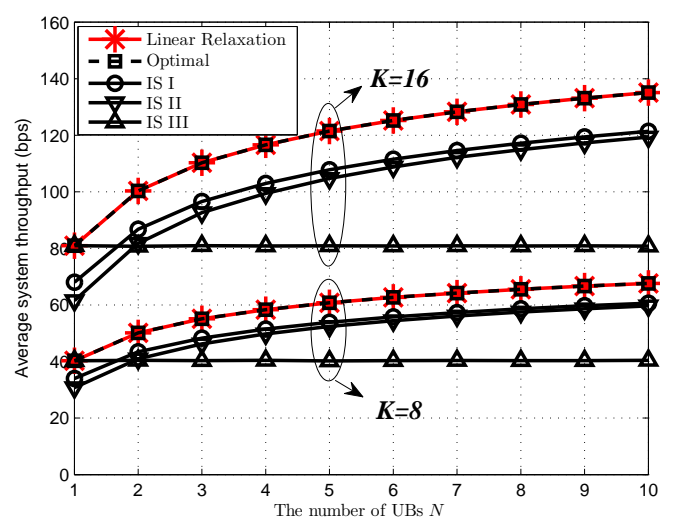

Fig. 3. Average sum rate vs the numbers of UGs $N$ for $K=8$ and $K=16$ when $\mu_{S R}=30 \mathrm{~dB}, \mu_{R D}=35 \mathrm{~dB}$ and $\mu_{R R}=5 \mathrm{~dB}$.

Meanwhile, it has also been verified that our proposed resource allocation algorithm is able to improve the system throughput of full-duplex relay-assisted D2D multicarrier systems.

\section{APPENDIX A \\ Proof of QuAsi-ConCAVITy of Problem (8)}

By the relation $\alpha(n, k)+\beta(n, k)=1$ given in (8), we can first substitute $\beta(n, k)=1-\alpha(n, k)$ into the expression of $R(n, k)$, so that the original optimization problem with two variables can be equivalently simplified to an optimization problem with a single variable, $\alpha(n, k)$. As a result, we can rewrite the power allocation problem as follows:

$$
\begin{aligned}
& \bar{R}(n, k)=\max _{\alpha(n, k)} R(n, k), \quad \forall n \in \mathcal{N}, k \in \mathcal{K} \\
& \text { s.t. } \quad \alpha(n, k) \in(0,1) .
\end{aligned}
$$

Therefore, what we only need to prove is that $R(n, k)$ is a quasi-concave function with respect to $\alpha(n, k)$. According to the fundamental properties of a quasi-concave function, what we need to prove is the conjecture infra [19]:

Conjecture 1:

$$
\begin{aligned}
\forall & a_{1}(n, k), a_{2}(n, k) \in(0,1) \text { and } \lambda \in(0,1), \\
\exists & \left.R(n, k)\right|_{\alpha(n, k)=\lambda a_{1}(n, k)+(1-\lambda) a_{2}(n, k)} \geq \\
& \min \left(\left.R(n, k)\right|_{\alpha(n, k)=\alpha_{1}(n, k)},\left.R(n, k)\right|_{\alpha(n, k)=\alpha_{2}(n, k)}\right)
\end{aligned}
$$

To prove the conjecture, we first propose two lemmas as follows. 
Lemma 1: For bounded $x \in\left(x_{\min }, x_{\max }\right)$, if $g(x)$ is a bounded, continuous and monotonically increasing function and $h(x)$ is a bounded, continuous and monotonically decreasing function, $f(x)=\min (g(x), h(x))$ will be quasi-concave.

Proof: See Appendix in [12].

Lemma 2: For bounded $x>0$, if $f(x)$ is a quasi-concave function with respect to $x$, then $t(x)=\log _{2}(1+f(x))$ will also be a quasi-concave function with respect to $x$.

Proof: Because $f(x)$ is a quasi-concave function with respect to $x$, we have the relation infra:

$$
f\left(\lambda x_{1}+(1-\lambda) x_{2}\right) \geq \min \left(f\left(x_{1}\right), f\left(x_{2}\right)\right), \forall \lambda \in(0,1) .
$$

Because $\log _{2}(\cdot)$ is a monotonically increasing function of the variable enclosed, we can further derive the relation below:

$$
\begin{aligned}
t\left(\lambda x_{1}+(1-\lambda) x_{2}\right) & =\log _{2}\left(1+f\left(\lambda x_{1}+(1-\lambda) x_{2}\right)\right) \\
& \geq \log _{2}\left(1+\min \left(f\left(x_{1}\right), f\left(x_{2}\right)\right)\right) .
\end{aligned}
$$

Again, because of the monotonicity of $\log _{2}(\cdot)$, the equality given below is obviously valid:

$$
\begin{aligned}
\log _{2}\left(1+\min \left(f\left(x_{1}\right), f\left(x_{2}\right)\right)\right) & =\min \left(\log _{2}\left(1+f\left(x_{1}\right)\right), \log _{2}\left(1+f\left(x_{2}\right)\right)\right) \\
& =\min \left(t\left(x_{1}\right), t\left(x_{2}\right)\right) .
\end{aligned}
$$

Substituting (15) into (14) yields

$$
t\left(\lambda x_{1}+(1-\lambda) x_{2}\right) \geq \min \left(t\left(x_{1}\right), t\left(x_{2}\right)\right), \forall \lambda \in(0,1) .
$$

Therefore, as the relation proved in (16), $t(x)=\log _{2}(1+f(x))$ is also a quasi-concave function with respect to $x$.

By Lemma 1 and Lemma 2, now we are ready to prove Conjecture 1. It can be easily found that $\Gamma_{S R}(n, k)$ is a monotonically increasing function of $\alpha(n, k)$, while $\Gamma_{R D}(n, k)$ is a monotonically decreasing function of $\alpha(n, k)$. Hence, we let $g(\alpha(n, k))=\Gamma_{S R}(n, k)$ and $h(\alpha(n, k))=\Gamma_{R D}(n, k)$. Therefore, by Lemma 1 , the equivalent end-to-end $\operatorname{SIR} \Gamma_{S R D}(n, k)=$ $\min \left(\Gamma_{S R}(n, k), \Gamma_{R D}(n, k)\right)$ is a quasi-concave function of $\alpha(n, k)$. Finally, by Lemma 2 , it is evident that the transmission rate $R(n, k)=\log _{2}\left(1+\Gamma_{S R D}(n, k)\right)$ is also a quasi-concave function with respect to $\alpha(n, k)$.

\section{APPENDIX B}

\section{Proof of THE Equivalence of Problems (9) AND (10)}

To prove the equivalence of the formulated MBIP problem and its linear relaxation, we express the feasible sets of both by matrices and then analyze their total unimodularity. We organize $w(n, k)$ in the set $\mathbf{w}=$ $(w(1,1), \cdots w(1, K), w(2,1), \cdots, w(N, K))^{T} \in\{0,1\}^{K N}$ and define

$$
\mathbf{B}:=\mathbf{1}_{N}^{T} \otimes \mathbf{I}_{K}=\underbrace{\left(\mathbf{I}_{K}, \mathbf{I}_{K}, \cdots, \mathbf{I}_{K}\right)}_{N} \in\{0,1\}^{K \times K N}
$$

where $\mathbf{x}_{Y}$ denotes a $Y \times 1$ vector of $x$ and $\mathbf{I}_{K}$ denotes a $K \times K$ identity matrix; $\otimes$ represents the Kronecker product; $(\cdot)^{T}$ represents the transpose of the matrix enclosed.

Hence, the feasible sets of (9) and (10) can be expressed by

$$
\mathcal{F}=\left\{\mathbf{w} \in\{0,1\}^{K N} \mid \mathbf{B w} \leq \mathbf{1}_{K}\right\} \text { and } \tilde{\mathcal{F}}=\left\{\tilde{\mathbf{w}} \in \mathbb{R}^{K N} \mid \mathbf{C} \tilde{\mathbf{w}} \leq \mathbf{u}\right\}
$$

where $\tilde{\mathbf{w}}$ is produced similarly as $\mathbf{w}$, but with elements $\tilde{w}(n, k)$, $\forall n \in \mathcal{N}, k \in \mathcal{K} ; \mathbf{C}:=\left(\mathbf{B}^{T}, \mathbf{I}_{K N},-\mathbf{I}_{K N}\right)^{T}$ and $\mathbf{u}:=$ $\left(\mathbf{1}_{K}^{T}, \mathbf{1}_{K N}^{T}, \mathbf{0}_{K N}^{T}\right)^{T}$.
Meanwhile, according to the definition of the total unimodularity, a matrix is totally unimodular if every square submatrix has determinant \pm 1 or 0 [20]. Therefore, by (17) and $\mathbf{C}:=$ $\left(\mathbf{B}^{T}, \mathbf{I}_{K N},-\mathbf{I}_{K N}\right)^{T}$, it is evident that $\mathbf{B}$ and $\mathbf{C}$ are totally unimodular. Also, because $\mathbf{u}$ is an integer vector, it can be proved by the integer optimization theory that the feasible set $\tilde{\mathcal{F}}$ has integral extreme points and thus the MBIP problem with the feasible set $\mathcal{F}$ is equivalent to the LP problem with the feasible set $\tilde{\mathcal{F}}$ This proved equivalence allows us to solve the NP-hard MBIP problem formulated in (9) by its linear relaxation formulated in (10), which will reduce the computational complexity from exponential time to polynomial time.

\section{REFERENCES}

[1] A. Asadi, Q. Wang, and V. Mancuso, "A survey on device-to-device communication in cellular networks," IEEE Commun. Surveys Tutorials, vol. 16, no. 4, pp. 1801-1819, 2014.

[2] J. Deng, A. A. Dowhuszko, R. Freij, and O. Tirkkonen, "Relay selection and resource allocation for D2D-relaying under uplink cellular power control," in Proc. IEEE Globecom, San Diego, CA, USA, Dec. 2015.

[3] B. Debaillie, D.-J. van den Broek, C. Lavin, B. van Liempd, E. Klumperink, C. Palacios, J. Craninckx, B. Nauta, and A. Parssinen, "Analog/RF solutions enabling compact full-duplex radios," IEEE J. Sel. Areas Commun., vol. 32, no. 9, pp. 1662-1673, Sept. 2014.

[4] M. Chung, M. S. Sim, J. Kim, D. K. Kim, and C. b. Chae, "Prototyping realtime full duplex radios," IEEE Commun. Mag., vol. 53, no. 9, pp. 56-63, Sept. 2015.

[5] G. Chen, Y. Gong, P. Xiao, and J. A. Chambers, "Physical layer network security in the full-duplex relay system," IEEE Trans. Inform. Forensics and Security, vol. 10, no. 3, pp. 574-583, Apr. 2015.

[6] T. Yang, R. Zhang, X. Cheng, and L. Yang, "Resource sharing for device-todevice communications underlaying full-duplex cellular networks," in Proc. IEEE ICCS, Macau, China, Nov. 2014.

[7] S. Ali, A. Ghazanfari, N. Rajatheva, and M. Latva-aho, "Effect of residual of self-interference in performance of full-duplex D2D communication," in Proc. IEEE 5GU, Levi, Finland, Nov. 2014.

[8] H. Bagheri, F. A. M. Bonomi, and M. Katz, "Spectral efficiency and throughput enhancement by full-duplex D2D communication in mobile clouds," in Proc. IEEE European Wireless Conference, May 2015.

[9] G. Zhang, K. Yang, P. Liu, and J. Wei, "Power allocation for full-duplex relaying-based D2D communication underlaying cellular networks," IEEE Trans. on Veh. Technol., vol. 64, no. 10, pp. 4911-4916, Oct. 2015.

[10] Z. Lin, Y. Li, S. Wen, Y. Gao, X. Zhang, and D. Yang, "Stochastic geometry analysis of achievable transmission capacity for relay-assisted device-todevice networks," in Proc. IEEE ICC, Sydney, Australia, June 2014.

[11] J. F. Shi, L. Tao, M. Chen, and Z. H. Yang, "Power control for relayassisted device-to-device communication underlaying cellular networks," in Proc. IEEE WCSP, Nanjing, China, Oct. 2015.

[12] S. Dang, G. Chen, and J. Coon, "Outage performance analysis of fullduplex relay-assisted device-to-device systems in uplink cellular networks," To appear IEEE Trans. on Veh. Technol., 2016.

[13] L. Liu, Y. Zhang, S. Liu, and Z. Zhang, "Power allocation optimization for D2D communication underlaying cognitive full duplex relay networks," in Proc. IEEE WiCOM, Shanghai, China, Sept. 2015.

[14] W. Cheng, X. Zhang, and H. Zhang, "Optimal power allocation for fullduplex D2D communications over wireless cellular networks," in Proc. GLOBECOM, Austin, TX, USA, Dec. 2014.

[15] R. M. Karp, "Reducibility among combinatorial problems," Complexity of computer computations, pp. 85-103, 1972.

[16] L. Wang and G. L. Stuber, "Pairing for resource sharing in cellular deviceto-device underlays," IEEE Network, vol. 30, no. 2, pp. 122-128, Mar. 2016.

[17] J. Jang and K. B. Lee, "Transmit power adaptation for multiuser OFDM systems," IEEE J. Sel. Areas Commun., vol. 21, no. 2, pp. 171-178, Feb. 2003.

[18] M. Al-Imari, M. Ghoraishi, and P. Xiao, "Radio resource allocation for full-duplex multicarrier wireless systems," in Proc. IEEE ISWCS, Brussels, Belgium, Aug. 2015.

[19] A. C. E. Kenneth J. Arrow, "Quasi-concave programming," Econometrica, vol. 29 , no. 4 , pp. $779-800,1961$.

[20] D. Bertsimas and R. Weismantel, Optimization over integers. Dynamic Ideas Belmont, 2005, vol. 13. 